\title{
A THEOREM ON PRODUCTIVE FUNCTIONS
}

\author{
T. G. McLAUGHLIN
}

It has been known for some time $[1, \text { p. } 47 \text {, footnote }]^{1}$ that an arbitrary productive set of natural numbers has $\boldsymbol{\aleph}_{0}$ mutually disjoint productive centers; however, so far as we know, no proof of this fact has ever been published. It may therefore be worthwhile to exhibit a very simple proof of a somewhat more strongly formulated result.

THEOREM. Let $\alpha$ be a productive set (resp., a set contraproductive under a total function); then, $\alpha$ admits $\boldsymbol{\aleph}_{0}$ productive (contraproductive) functions having mutually disjoint ranges.

PRoof. Myhill has proved that any productive set is productive under a 1-1 total function. A corresponding result is easily established for any contraproductive set having at least one total contraproductive function. Letting $h(x, y)$ be the recursive function of $[2$, Theorem 2.4 , p. 69], consider the sequence $s_{0}=h(0,0), s_{1}=h\left(1, s_{0}\right), s_{2}=h\left(0, s_{1}\right)$, $s_{3}=h\left(1, s_{2}\right), s_{4}=h\left(2, s_{3}\right)$, and so on. This sequence gives $\boldsymbol{\aleph}_{0}$ mutually disjoint effective enumerations (e.g., $s_{0}, s_{1}, s_{4}, \cdots$ ) of indices of all the r.e. sets. Let $\psi_{i}(x), 0 \leqq i<\omega$, be recursive functions providing these disjoint enumerations. Then, if $p(x)$ is any 1-1 productive (contraproductive) function for $\alpha$, it is straightforward to verify that (i) each function $p \circ \psi_{i}$ is also a productive (contraproductive) function for $\alpha$, and (ii) the $p \circ \psi_{i}$ 's have mutually disjoint ranges.

Added in proof. Since this note was submitted, the author has observed the following result: any contraproductive set admitting a total contraproductive function is actually productive. Thus, in particular, the hypothesis of the theorem does not cover the case of the complement of a simple or mesoic r.e. set; nevertheless, the conclusion of the theorem (for contraproductivity) does hold for such sets.

\section{REFERENCES}

1. J. C. E. Dekker and J. Myhill, Some theorems on classes of recursively enumerable sets, Trans. Amer. Math. Soc. 89 (1958), 25-59.

2. R. M. Smullyan, Theory of formal systems, Princeton Univ. Press, Princeton, N. J., 1961.

University of California, Los Angeles

Received by the editors March 30, 1962.

${ }^{1}$ We thank Professor Dekker for drawing our attention to this reference. 